Supporting Information

\title{
Two Series of Lanthanide Coordination Polymers with 2-Methylenesuccinate: Magnetic Refrigerant, Slow Magnetic Relaxation, and Luminescent Properties
}

Zhong-Yi Li, Bin Zhai, *Su-Zhi Li, Guang-Xiu Cao, Fu-Qiang Zhang, Xiang-Fei Zhang, Fu-Li Zhang,and Chi Zhang

College of Chemistry and Chemical Engineering, Shangqiu Normal University, Shangqiu 476000, P. R. China 
Table S1. Selected bond lengths $(\AA)$ and angles $\left(^{\circ}\right)$ for $\mathbf{1}$.

\begin{tabular}{|c|c|c|c|}
\hline $\mathrm{Gd}(1)-\mathrm{O}(3)$ & $2.303(3)$ & $\mathrm{Gd}(1)-\mathrm{O}(1)$ & $2.434(3)$ \\
\hline $\mathrm{Gd}(1)-\mathrm{O}(4) \# 1$ & $2.357(3)$ & $\mathrm{Gd}(1)-\mathrm{O}(5)$ & $2.467(3)$ \\
\hline $\mathrm{Gd}(1)-\mathrm{O}(9)$ & $2.362(3)$ & $\mathrm{Gd}(1)-\mathrm{O}(1) \# 2$ & $2.740(3)$ \\
\hline $\mathrm{Gd}(1)-\mathrm{O}(8)$ & $2.397(3)$ & $\mathrm{Gd}(1)-\mathrm{O}(6)$ & $2.745(4)$ \\
\hline $\mathrm{Gd}(1)-\mathrm{O}(2) \# 2$ & $2.432(3)$ & & \\
\hline $\mathrm{O}(3)-\mathrm{Gd}(1)-\mathrm{O}(4) \# 1$ & $157.63(11)$ & $\mathrm{O}(2) \# 2-\mathrm{Gd}(1)-\mathrm{O}(5)$ & $76.20(11)$ \\
\hline $\mathrm{O}(3)-\mathrm{Gd}(1)-\mathrm{O}(9)$ & $83.28(12)$ & $\mathrm{O}(1)-\mathrm{Gd}(1)-\mathrm{O}(5)$ & $141.13(11)$ \\
\hline $\mathrm{O}(4) \# 1-\mathrm{Gd}(1)-\mathrm{O}(9)$ & $86.35(13)$ & $\mathrm{O}(3)-\mathrm{Gd}(1)-\mathrm{O}(1) \# 2$ & $69.19(9)$ \\
\hline $\mathrm{O}(3)-\mathrm{Gd}(1)-\mathrm{O}(8)$ & $84.79(12)$ & $\mathrm{O}(4) \# 1-\mathrm{Gd}(1)-\mathrm{O}(1) \# 2$ & $129.97(10)$ \\
\hline $\mathrm{O}(4) \# 1-\mathrm{Gd}(1)-\mathrm{O}(8)$ & $92.85(12)$ & $\mathrm{O}(9)-\mathrm{Gd}(1)-\mathrm{O}(1) \# 2$ & $136.06(11)$ \\
\hline $\mathrm{O}(9)-\mathrm{Gd}(1)-\mathrm{O}(8)$ & $145.66(11)$ & $\mathrm{O}(8)-\mathrm{Gd}(1)-\mathrm{O}(1) \# 2$ & $66.79(10)$ \\
\hline $\mathrm{O}(3)-\mathrm{Gd}(1)-\mathrm{O}(2) \# 2$ & $118.54(10)$ & $\mathrm{O}(2) \# 2-\mathrm{Gd}(1)-\mathrm{O}(1) \# 2$ & $49.46(9)$ \\
\hline $\mathrm{O}(4) \# 1-\mathrm{Gd}(1)-\mathrm{O}(2) \# 2$ & $82.06(10)$ & $\mathrm{O}(1)-\mathrm{Gd}(1)-\mathrm{O}(1) \# 2$ & $127.13(4)$ \\
\hline $\mathrm{O}(9)-\mathrm{Gd}(1)-\mathrm{O}(2) \# 2$ & $137.82(11)$ & $\mathrm{O}(5)-\mathrm{Gd}(1)-\mathrm{O}(1) \# 2$ & $66.33(10)$ \\
\hline $\mathrm{O}(8)-\mathrm{Gd}(1)-\mathrm{O}(2) \# 2$ & $75.62(11)$ & $\mathrm{O}(3)-\mathrm{Gd}(1)-\mathrm{O}(6)$ & $122.85(10)$ \\
\hline $\mathrm{O}(3)-\mathrm{Gd}(1)-\mathrm{O}(1)$ & $76.47(10)$ & $\mathrm{O}(4) \# 1-\mathrm{Gd}(1)-\mathrm{O}(6)$ & $71.17(11)$ \\
\hline $\mathrm{O}(4) \# 1-\mathrm{Gd}(1)-\mathrm{O}(1)$ & $81.70(10)$ & $\mathrm{O}(9)-\mathrm{Gd}(1)-\mathrm{O}(6)$ & $70.14(12)$ \\
\hline $\mathrm{O}(9)-\mathrm{Gd}(1)-\mathrm{O}(1)$ & $74.67(11)$ & $\mathrm{O}(8)-\mathrm{Gd}(1)-\mathrm{O}(6)$ & $141.46(12)$ \\
\hline $\mathrm{O}(8)-\mathrm{Gd}(1)-\mathrm{O}(1)$ & $71.26(10)$ & $\mathrm{O}(2) \# 2-\mathrm{Gd}(1)-\mathrm{O}(6)$ & $67.73(11)$ \\
\hline $\mathrm{O}(2) \# 2-\mathrm{Gd}(1)-\mathrm{O}(1)$ & $142.10(10)$ & $\mathrm{O}(1)-\mathrm{Gd}(1)-\mathrm{O}(6)$ & $136.31(11)$ \\
\hline $\mathrm{O}(3)-\mathrm{Gd}(1)-\mathrm{O}(5)$ & $76.79(11)$ & $\mathrm{O}(5)-\mathrm{Gd}(1)-\mathrm{O}(6)$ & $48.14(10)$ \\
\hline $\mathrm{O}(4) \# 1-\mathrm{Gd}(1)-\mathrm{O}(5)$ & $119.31(11)$ & $\mathrm{O}(1) \# 2-\mathrm{Gd}(1)-\mathrm{O}(6)$ & $96.38(10)$ \\
\hline $\mathrm{O}(9)-\mathrm{Gd}(1)-\mathrm{O}(5)$ & $74.63(12)$ & $\mathrm{Gd}(1)-\mathrm{O}(1)-\mathrm{Gd}(1) \# 1$ & $129.76(11)$ \\
\hline $\mathrm{O}(8)-\mathrm{Gd}(1)-\mathrm{O}(5)$ & $133.06(11)$ & & \\
\hline
\end{tabular}

Symmetry codes: $\# 1-\mathrm{x}+1 / 2, \mathrm{y}+1 / 2,-\mathrm{z}+1 / 2 ; \# 2-\mathrm{x}+1 / 2, \mathrm{y}-1 / 2,-\mathrm{z}+1 / 2$. 
Table S2. Selected bond lengths $(\AA)$ and angles $\left({ }^{\circ}\right)$ for 4.

\begin{tabular}{|c|c|c|c|}
\hline $\mathrm{Gd}(1)-\mathrm{O}(12)$ & $2.456(3)$ & $\mathrm{Gd}(1)-\mathrm{O}(9) \# 3$ & $2.473(3)$ \\
\hline $\mathrm{Gd}(1)-\mathrm{O}(13) \# 2$ & $2.490(3)$ & $\mathrm{Gd}(2)-\mathrm{O}(10)$ & $2.341(3)$ \\
\hline $\mathrm{Gd}(1)-\mathrm{O}(3) \# 1$ & $2.421(3)$ & $\mathrm{Gd}(2)-\mathrm{O}(11) \# 5$ & $2.435(3)$ \\
\hline $\mathrm{Gd}(1)-\mathrm{O}(4)$ & $2.387(3)$ & $\mathrm{Gd}(2)-\mathrm{O}(14)$ & $2.358(3)$ \\
\hline $\mathrm{Gd}(1)-\mathrm{O}(4) \# 1$ & $2.715(3)$ & $\mathrm{Gd}(2)-\mathrm{O}(15)$ & $2.384(3)$ \\
\hline $\mathrm{Gd}(1)-\mathrm{O}(5)$ & $2.412(3)$ & $\mathrm{Gd}(2)-\mathrm{O}(16)$ & $2.387(3)$ \\
\hline $\mathrm{Gd}(1)-\mathrm{O}(6)$ & $2.313(3)$ & $\mathrm{Gd}(2)-\mathrm{O}(7)$ & $2.384(3)$ \\
\hline $\mathrm{Gd}(1)-\mathrm{O}(8) \# 3$ & $2.462(3)$ & $\mathrm{O}(1)-\mathrm{Gd}(2) \# 4$ & $2.594(3)$ \\
\hline $\mathrm{O}(2)-\mathrm{Gd}(2) \# 4$ & $2.435(3)$ & & \\
\hline $\mathrm{O}(6)-\mathrm{Gd}(1)-\mathrm{O}(3) \# 1$ & 79.92(11) & $\mathrm{O}(13) \# 2-\mathrm{Gd}(1)-\mathrm{O}(4) \# 1$ & $73.29(11)$ \\
\hline $\mathrm{O}(4)-\mathrm{Gd}(1)-\mathrm{O}(3) \# 1$ & $116.05(10)$ & $\mathrm{O}(10)-\mathrm{Gd}(2)-\mathrm{O}(7)$ & $85.33(11)$ \\
\hline $\mathrm{O}(5)-\mathrm{Gd}(1)-\mathrm{O}(3) \# 1$ & $78.07(11)$ & $\mathrm{O}(14)-\mathrm{Gd}(2)-\mathrm{O}(7)$ & $68.46(10)$ \\
\hline $\mathrm{O}(6)-\mathrm{Gd}(1)-\mathrm{O}(12) \# 2$ & $80.37(10)$ & $\mathrm{O}(10)-\mathrm{Gd}(2)-\mathrm{O}(15)$ & $158.87(12)$ \\
\hline $\mathrm{O}(4)-\mathrm{Gd}(1)-\mathrm{O}(12) \# 2$ & $127.12(11)$ & $\mathrm{O}(14)-\mathrm{Gd}(2)-\mathrm{O}(15)$ & $85.94(12)$ \\
\hline $\mathrm{O}(5)-\mathrm{Gd}(1)-\mathrm{O}(12) \# 2$ & $148.64(11)$ & $\mathrm{O}(7)-\mathrm{Gd}(2)-\mathrm{O}(15)$ & $75.29(12)$ \\
\hline $\mathrm{O}(3) \# 1-\mathrm{Gd}(1)-\mathrm{O}(12) \# 2$ & $74.44(11)$ & $\mathrm{O}(10)-\mathrm{Gd}(2)-\mathrm{O}(16)$ & 81.81(11) \\
\hline $\mathrm{O}(6)-\mathrm{Gd}(1)-\mathrm{O}(8) \# 3$ & $76.57(11)$ & $\mathrm{O}(14)-\mathrm{Gd}(2)-\mathrm{O}(16)$ & $143.13(11)$ \\
\hline $\mathrm{O}(4)-\mathrm{Gd}(1)-\mathrm{O}(8) \# 3$ & $77.39(10)$ & $\mathrm{O}(7)-\mathrm{Gd}(2)-\mathrm{O}(16)$ & $74.66(11)$ \\
\hline $\mathrm{O}(5)-\mathrm{Gd}(1)-\mathrm{O}(8) \# 3$ & $74.91(10)$ & $\mathrm{O}(15)-\mathrm{Gd}(2)-\mathrm{O}(16)$ & $84.97(12)$ \\
\hline $\mathrm{O}(3) \# 1-\mathrm{Gd}(1)-\mathrm{O}(8) \# 3$ & $146.65(10)$ & $\mathrm{O}(10)-\mathrm{Gd}(2)-\mathrm{O}(11) \# 5$ & $113.80(11)$ \\
\hline $\mathrm{O}(12) \# 2-\mathrm{Gd}(1)-\mathrm{O}(8) \# 3$ & $123.55(10)$ & $\mathrm{O}(14)-\mathrm{Gd}(2)-\mathrm{O}(11) \# 5$ & $140.23(11)$ \\
\hline $\mathrm{O}(6)-\mathrm{Gd}(1)-\mathrm{O}(9) \# 3$ & $83.01(11)$ & $\mathrm{O}(7)-\mathrm{Gd}(2)-\mathrm{O}(11) \# 5$ & $137.41(11)$ \\
\hline $\mathrm{O}(4)-\mathrm{Gd}(1)-\mathrm{O}(9) \# 3$ & $92.84(11)$ & $\mathrm{O}(15)-\mathrm{Gd}(2)-\mathrm{O}(11) \# 5$ & $76.95(11)$ \\
\hline $\mathrm{O}(5)-\mathrm{Gd}(1)-\mathrm{O}(9) \# 3$ & $127.27(10)$ & $\mathrm{O}(16)-\mathrm{Gd}(2)-\mathrm{O}(11) \# 5$ & $71.29(11)$ \\
\hline $\mathrm{O}(3) \# 1-\mathrm{Gd}(1)-\mathrm{O}(9) \# 3$ & $146.20(10)$ & $\mathrm{O}(10)-\mathrm{Gd}(2)-\mathrm{O}(2) \# 6$ & $72.26(10)$ \\
\hline $\mathrm{O}(12) \# 2-\mathrm{Gd}(1)-\mathrm{O}(9) \# 3$ & $74.07(11)$ & $\mathrm{O}(14)-\mathrm{Gd}(2)-\mathrm{O}(2) \# 6$ & $79.13(10)$ \\
\hline $\mathrm{O}(8) \# 3-\mathrm{Gd}(1)-\mathrm{O}(9) \# 3$ & $52.59(9)$ & $\mathrm{O}(7)-\mathrm{Gd}(2)-\mathrm{O}(2) \# 6$ & $138.70(11)$ \\
\hline $\mathrm{O}(6)-\mathrm{Gd}(1)-\mathrm{O}(13) \# 2$ & $132.10(10)$ & $\mathrm{O}(15)-\mathrm{Gd}(2)-\mathrm{O}(2) \# 6$ & $128.33(12)$ \\
\hline $\mathrm{O}(4)-\mathrm{Gd}(1)-\mathrm{O}(13) \# 2$ & $76.38(11)$ & $\mathrm{O}(16)-\mathrm{Gd}(2)-\mathrm{O}(2) \# 6$ & $132.76(10)$ \\
\hline $\mathrm{O}(5)-\mathrm{Gd}(1)-\mathrm{O}(13) \# 2$ & $141.07(11)$ & $\mathrm{O}(11) \# 5-\mathrm{Gd}(2)-\mathrm{O}(2) \# 6$ & $83.85(10)$ \\
\hline $\mathrm{O}(3) \# 1-\mathrm{Gd}(1)-\mathrm{O}(13) \# 2$ & $86.45(12)$ & $\mathrm{O}(10)-\mathrm{Gd}(2)-\mathrm{O}(1) \# 6$ & $123.61(10)$ \\
\hline $\mathrm{O}(12) \# 2-\mathrm{Gd}(1)-\mathrm{O}(13) \# 2$ & $51.73(10)$ & $\mathrm{O}(14)-\mathrm{Gd}(2)-\mathrm{O}(1) \# 6$ & $72.62(11)$ \\
\hline $\mathrm{O}(8) \# 3-\mathrm{Gd}(1)-\mathrm{O}(13) \# 2$ & $126.90(11)$ & $\mathrm{O}(7)-\mathrm{Gd}(2)-\mathrm{O}(1) \# 6$ & $133.06(10)$ \\
\hline $\mathrm{O}(9) \# 3-\mathrm{Gd}(1)-\mathrm{O}(13) \# 2$ & $83.68(11)$ & $\mathrm{O}(15)-\mathrm{Gd}(2)-\mathrm{O}(1) \# 6$ & $76.79(12)$ \\
\hline $\mathrm{O}(6)-\mathrm{Gd}(1)-\mathrm{O}(4) \# 1$ & $124.72(10)$ & $\mathrm{O}(16)-\mathrm{Gd}(2)-\mathrm{O}(1) \# 6$ & $138.71(11)$ \\
\hline $\mathrm{O}(4)-\mathrm{Gd}(1)-\mathrm{O}(4) \# 1$ & $66.21(11)$ & $\mathrm{O}(11) \# 5-\mathrm{Gd}(2)-\mathrm{O}(1) \# 6$ & $68.64(10)$ \\
\hline $\mathrm{O}(5)-\mathrm{Gd}(1)-\mathrm{O}(4) \# 1$ & $69.39(10)$ & $\mathrm{O}(2) \# 6-\mathrm{Gd}(2)-\mathrm{O}(1) \# 6$ & $51.54(9)$ \\
\hline $\mathrm{O}(3) \# 1-\mathrm{Gd}(1)-\mathrm{O}(4) \# 1$ & $49.87(9)$ & $\mathrm{Gd}(1)-\mathrm{O}(4)-\mathrm{Gd}(1) \# 1$ & $113.79(11)$ \\
\hline
\end{tabular}

Symmetry codes: \#1 -x+1, -y, -z+1; \#2 -x, -y, -z; \#3 -x+1, -y+1, -z+1; \#4 x+1, y, z+1; \#5 -x, -y+1, -z; \#6 x-1, y, z-1. 


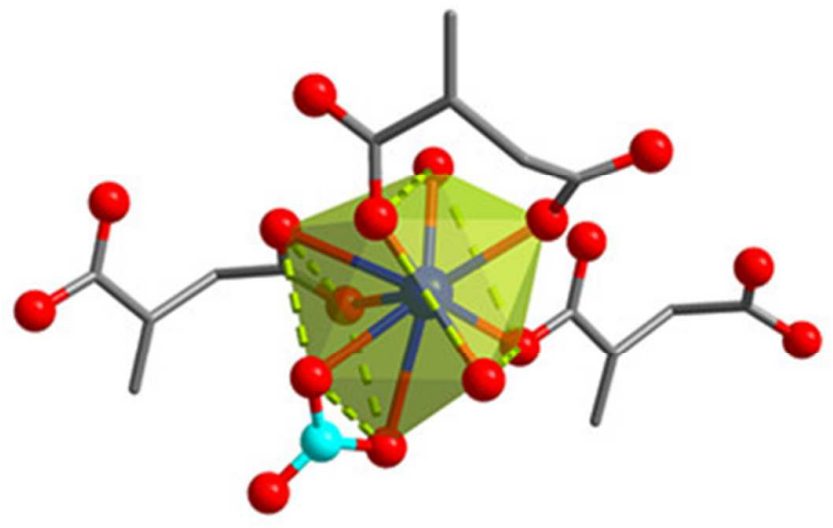

Figure S1. Coordination environment of $\mathrm{Gd}^{3+}$ ion of $\mathbf{1}$.

;

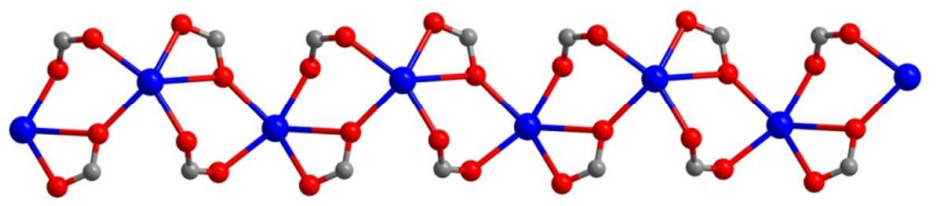

Figure S2. The 1D chain-shaped building units $\left[\mathrm{Gd}_{2}\left(\mathrm{CO}_{2}\right)_{4}\right]_{\mathrm{n}}$ constructed from the adjacent $\mathrm{Gd}^{3+}$ ions and carboxyl groups of the $\mathrm{H}_{2} \mathrm{MSA}$ ligands in $\mathbf{1}$.

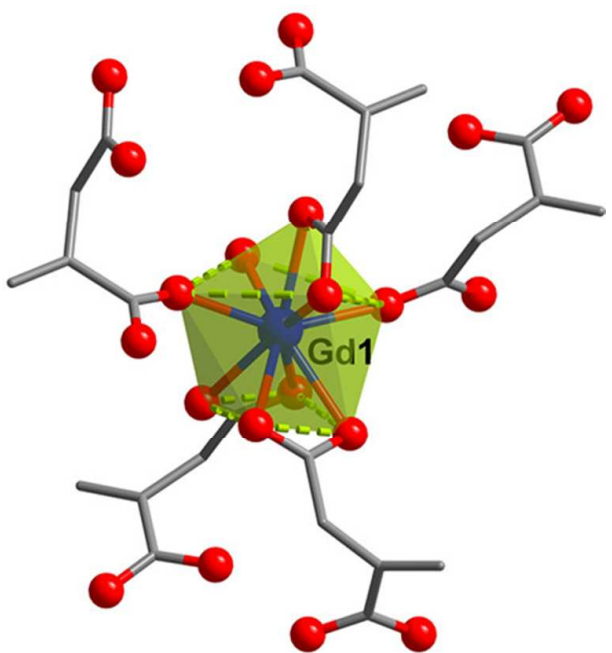

(a)

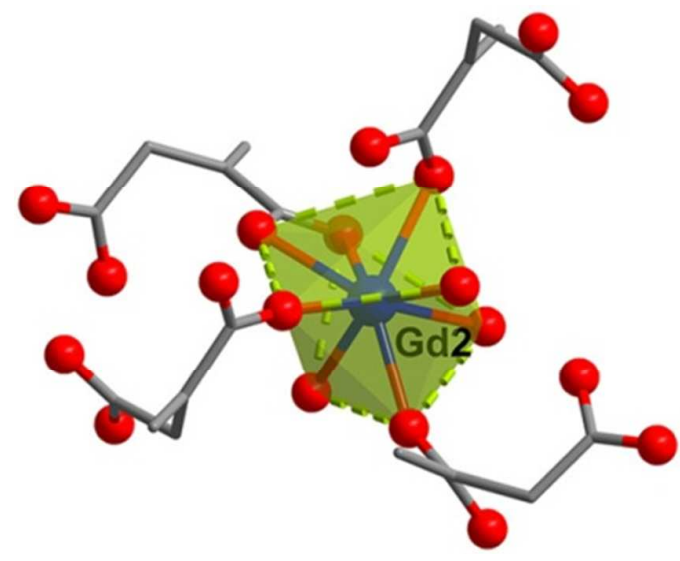

(b)

Figure S3. Coordination environment of $\mathrm{Gd}^{3+}$ ion in $\mathbf{4}$, (a) for $\mathrm{Gd} 1$ and (b) for $\mathrm{Gd} 2$. 


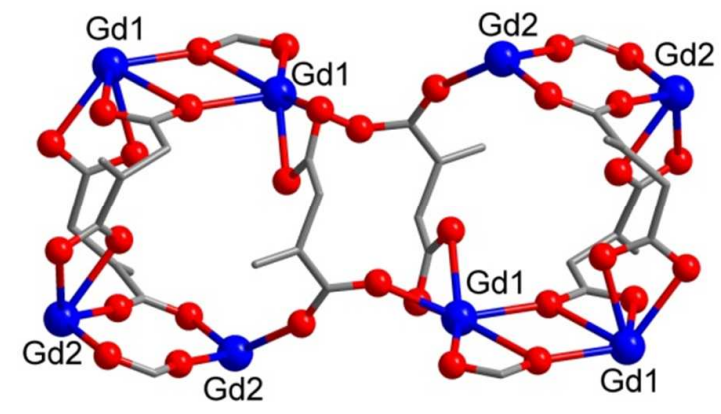

Figure S4. View of the connection of the neighboring 1D $\left[\mathrm{Gd}_{4}\left(\mathrm{CO}_{2}\right)_{6}\right]_{\mathrm{n}}$ units.

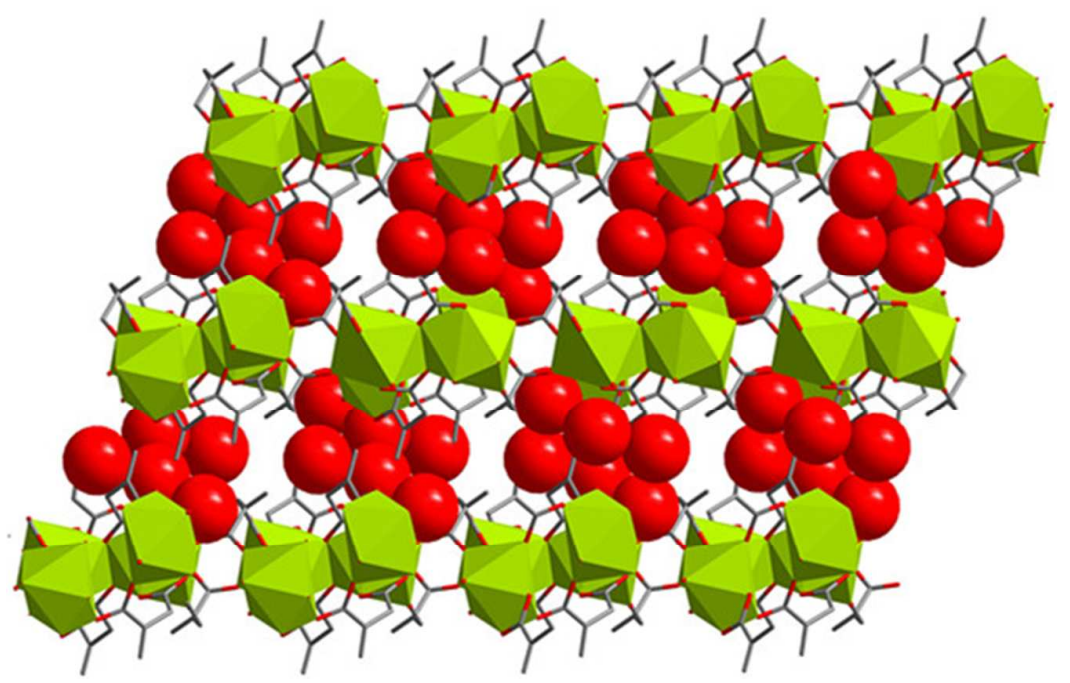

Figure S5. Packing View of the 2D structures viewed along [111] axis. The free water molecules are shown in space-filling mode. The hydrogen atoms have been omitted for clarity.

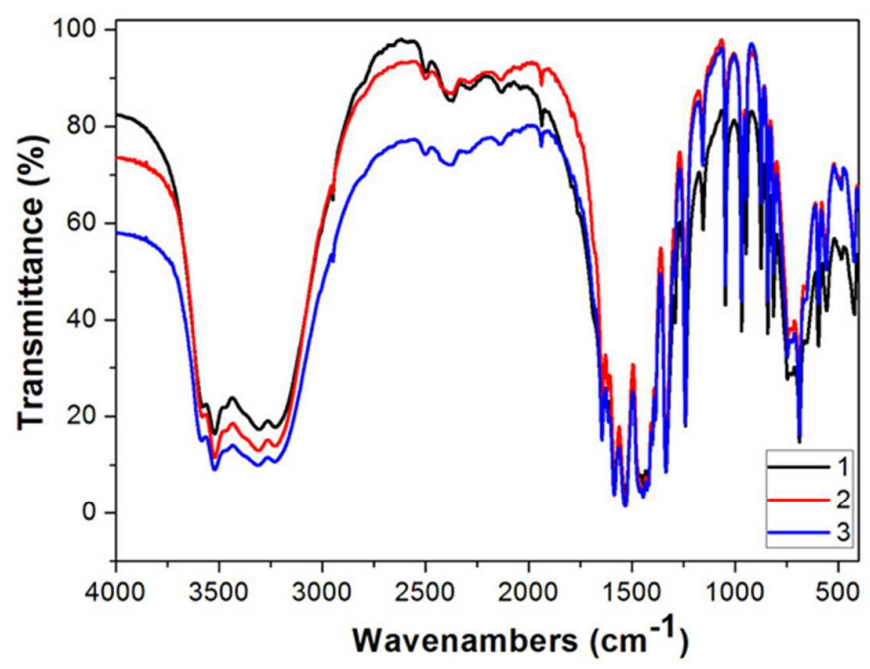

Figure S6. The IR spectra of 1-3. 


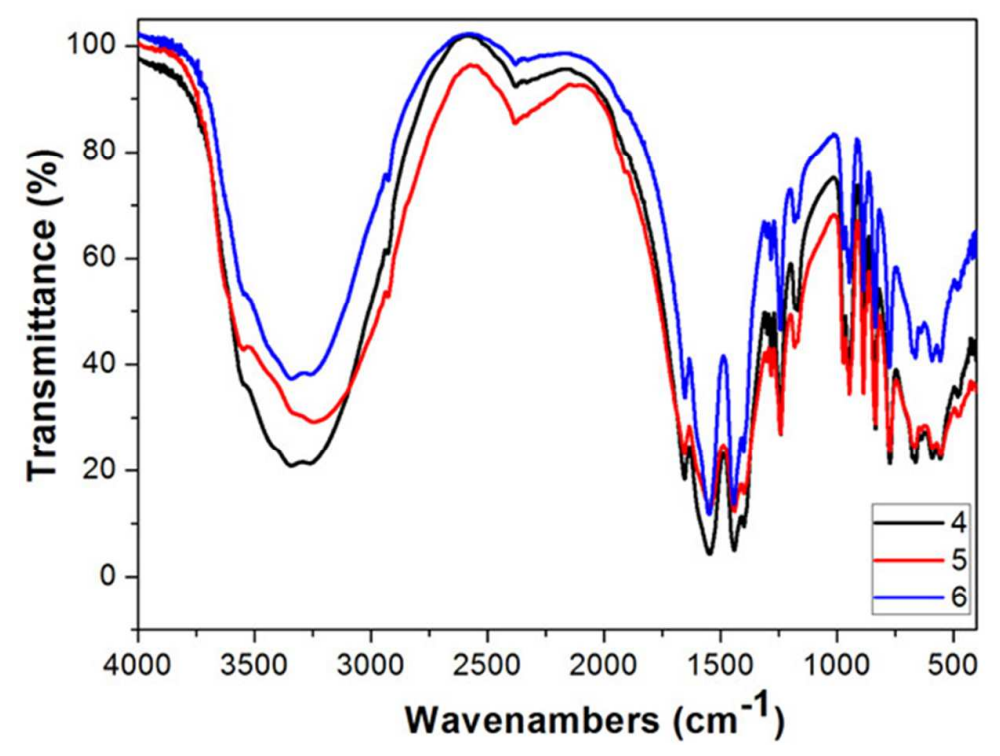

Figure S7. The IR spectra of 4-6.

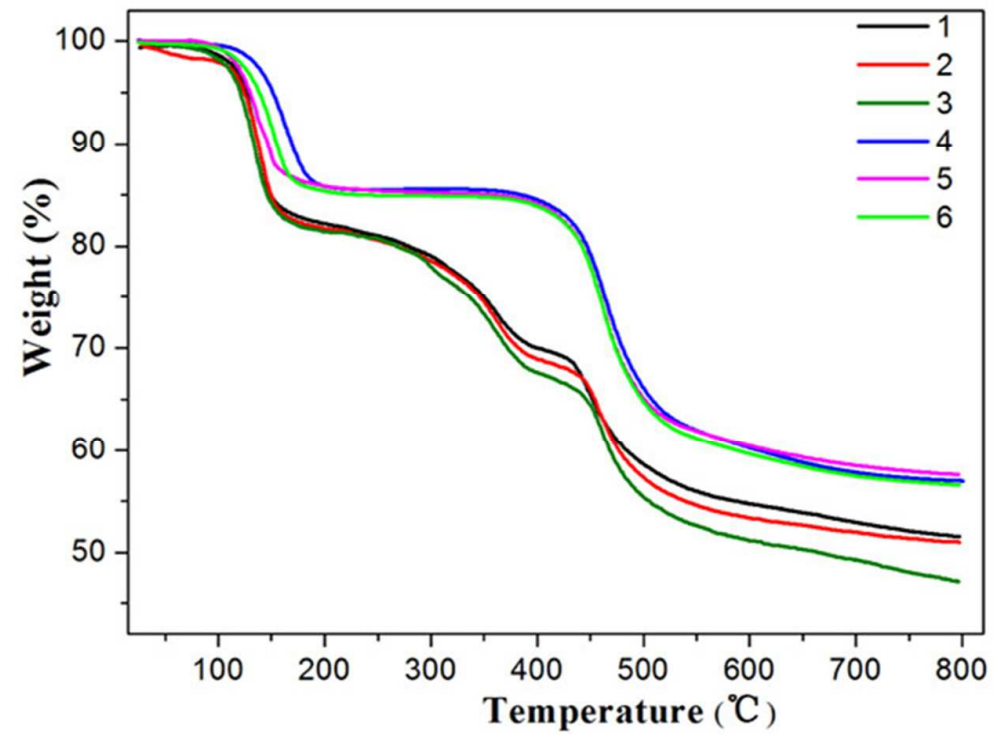

Figure S8. TG curves for $\mathbf{1}-\mathbf{6}$ in a nitrogen atmosphere $\left(10^{\circ} \mathrm{C} / \mathrm{min}\right)$. 


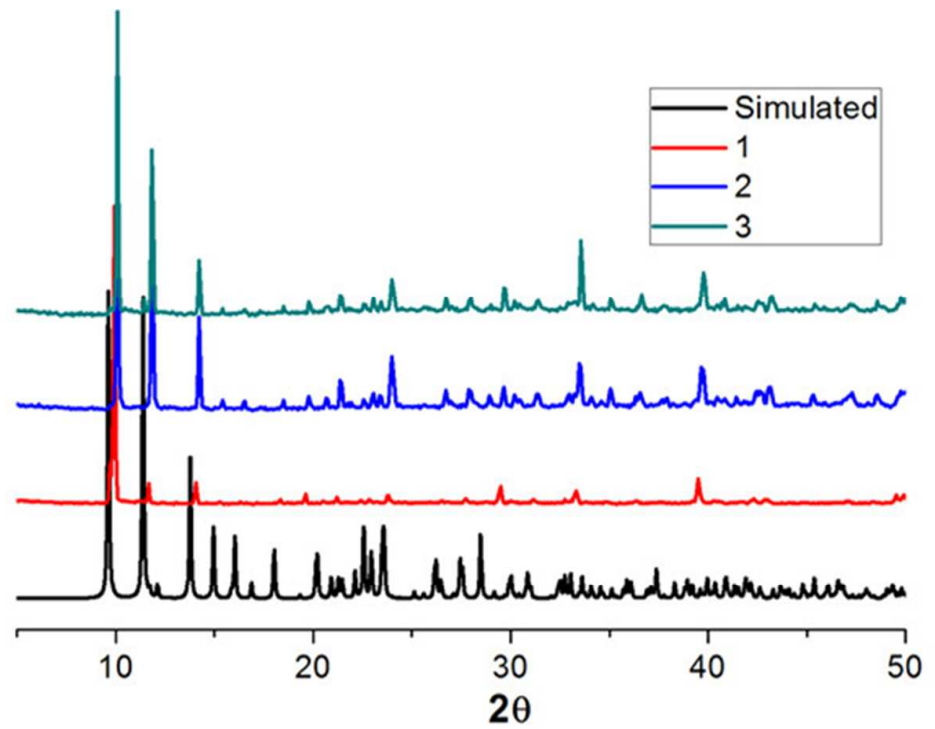

Figure S9. Powder X-ray diffraction patterns of 1-3.

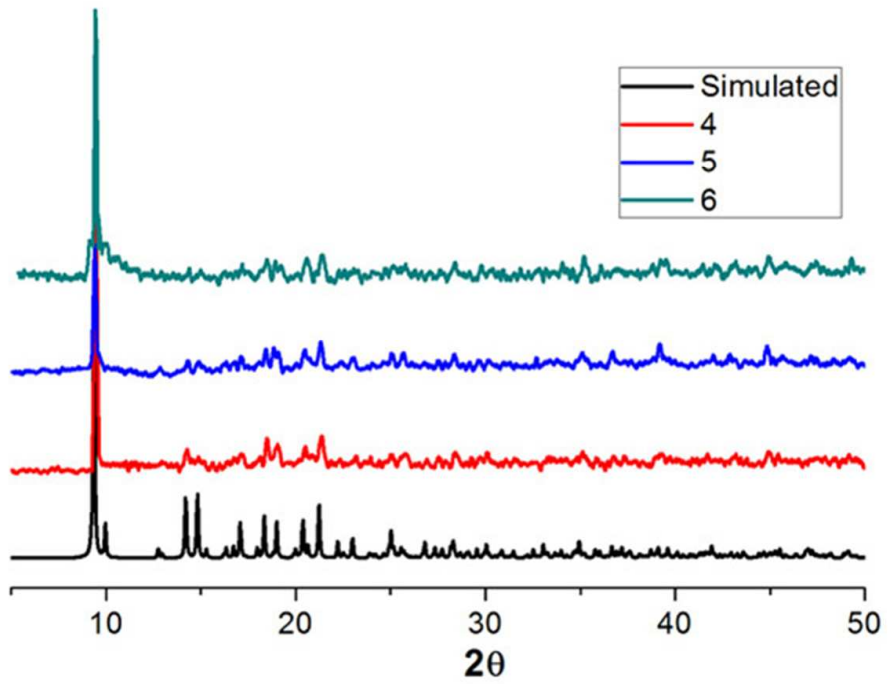

Figure S10. Powder X-ray diffraction patterns of 4-6. 


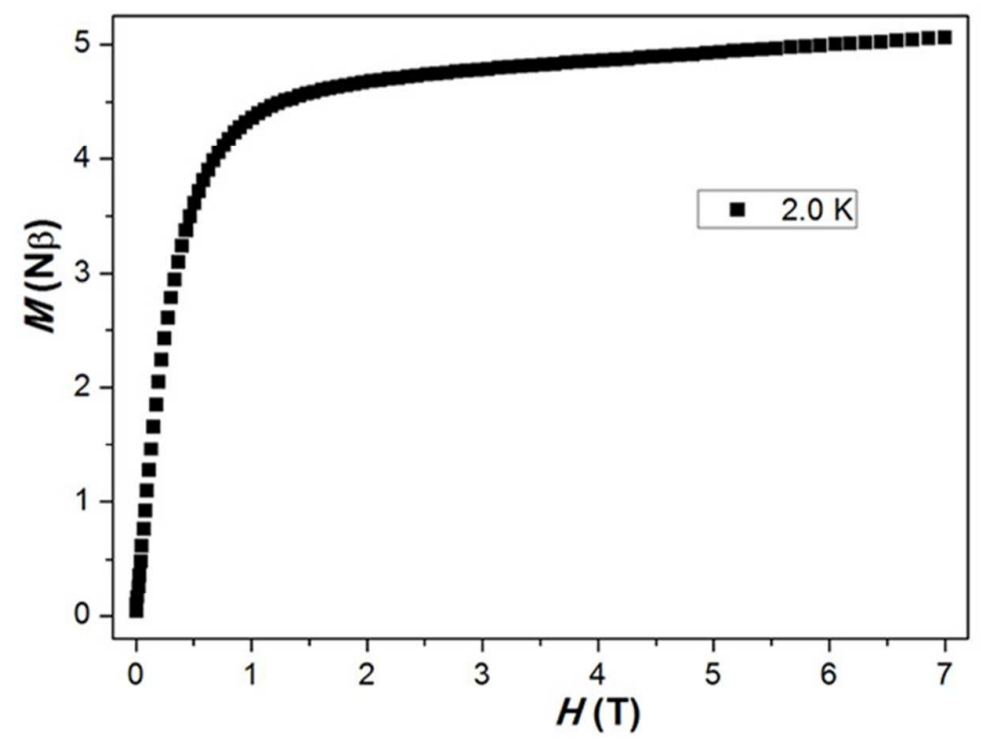

Figure S11. Field dependence of the magnetization of 3 at $2.0 \mathrm{~K}$.

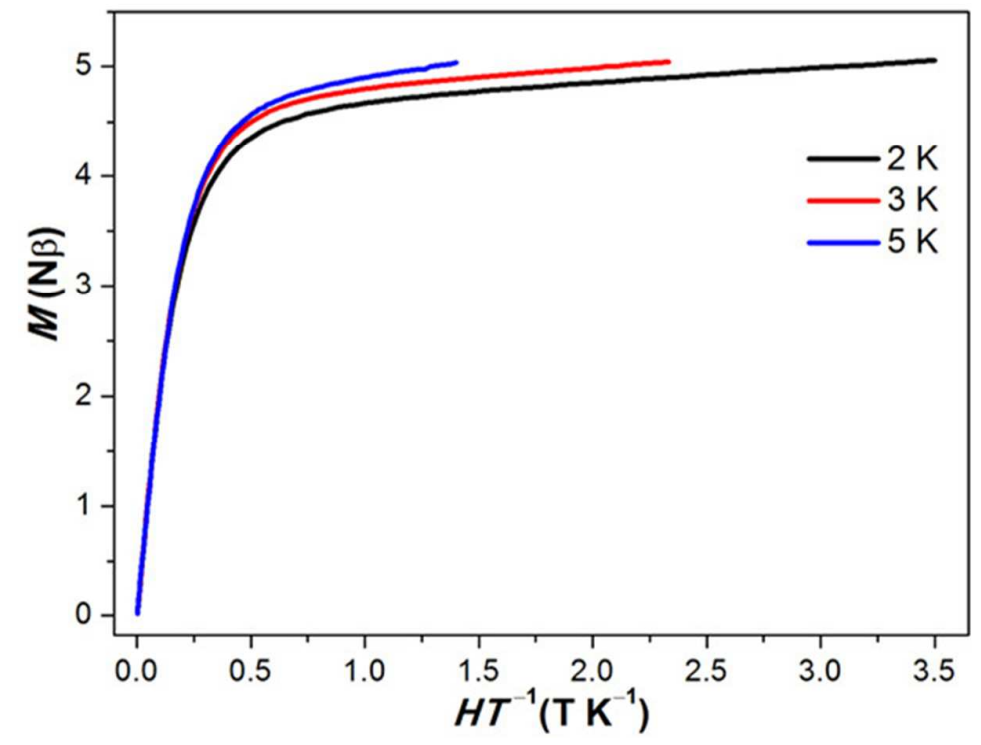

Figure S12. $M$ versus $H / T$ plots at different temperatures for 3 . 


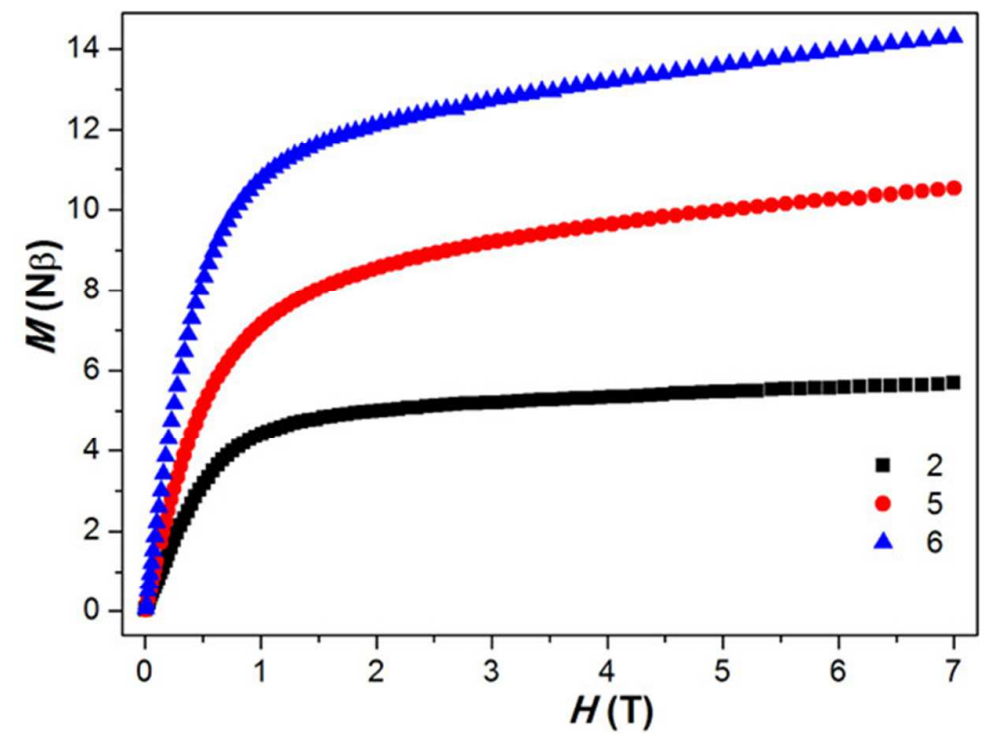

Figure S13. Field dependence of the magnetization of 2,5 and $\mathbf{6}$ at $2.0 \mathrm{~K}$.

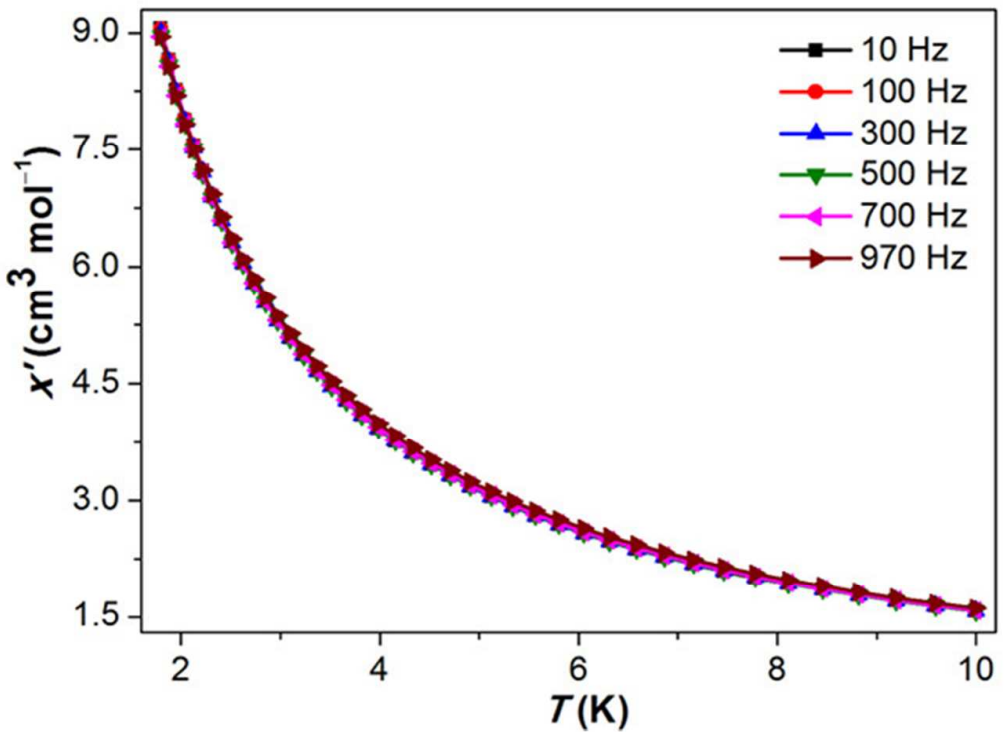

Figure S14. Temperature dependence of the in-phase $\left(\chi^{\prime}\right)$ ac susceptibility for 3 at the indicated frequencies and in the zero dc field. 